\title{
Comparison of performance between Fast Track Diagnostics Respiratory Kit and the CDC Global Reference Laboratory for Influenza rRT-PCR panel for detection of influenza A and B
}

Jennifer Milucky ${ }^{1}$, Assana Cisse ${ }^{2}$, Abdoul Ilboudo ${ }^{3}$, Jessica Waller ${ }^{1}$, Brice Bicaba ${ }^{4}$, Isaïe Médah $^{5}$, Sara Mirza ${ }^{1}$, Cynthia Whitney $^{1}$, and Zekiba Tarnagda ${ }^{6}$

${ }^{1}$ Centers for Disease Control and Prevention

${ }^{2}$ Institut de Recherche en Sciences de la Santé

${ }^{3}$ Institut de Recherche en Sciences de la Santé, Centre National de Référence pour la Grippe

${ }^{4}$ Direction de la Protection de la Santé de la Population

${ }^{5}$ Direction de la lutte contre la maladie (DLM), Ministère de la santé

${ }^{6}$ Institut de recherche en Sciences de la santé (IRSS)

August 4, 2020

\begin{abstract}
Background Reliable diagnostics are key to identifying influenza infections. Our objectives were to describe detection of influenza among severe acute respiratory infection (SARI) cases, to compare test results from the FTD-33 kit for influenza detection to the Centers for Disease Control (CDC) human influenza virus detection and characterization panel, and to assess seasonality of influenza in Burkina Faso. Methods: Nasopharyngeal and oropharyngeal specimens from SARI cases (hospitalized patients with fever, cough, and onset in the previous 10 days) were tested using the FTD-33 kit and the CDC rRT-PCR influenza assays. We assessed sensitivity and specificity of the FTD-33 kit for detecting Influenza A, Influenza B, and the influenza A(H1N1)pdm09 strain using the CDC human influenza rRT-PCR panel as the gold standard. Results: From December 2016 to February 2019, 1706 SARI cases were identified, 1,511 specimens were tested, and 211 were positive for influenza A (14.0\%) and 100 for influenza B (6.6\%) by either assay. Higher influenza circulation occurred between November and April with varying peaks of influenza A and B. Sensitivity of the FTD-33 assay was $91.9 \%$ for influenza A, $95.7 \%$ for influenza B, and $93.8 \%$ for A(H1N1)pdm09 subtype. Specificity was over $99 \%$ for all three tests. Conclusions: Our study indicates that Burkina Faso has one peak of influenza each year which is similar to the Northern Hemisphere and differs from other countries in West Africa. We found high concordance of influenza results between the two assays indicating FTD-33 can be used to reliably detect influenza among SARI cases.
\end{abstract}

\section{Hosted file}

Manuscript with tables 23July2020.docx available at https://authorea.com/users/348596/ articles/473819-comparison-of-performance-between-fast-track-diagnostics-respiratorykit-and-the-cdc-global-reference-laboratory-for-influenza-rrt-pcr-panel-for-detectionof-influenza-a-and-b 